\title{
Defining treatment thresholds for neonatal hypoglycaemia-how low is too low?
}

$\mathrm{M}$

any newborn babies experience hypoglycaemia, which can lead to neurological impairment. Furthermore, although neonatal hypoglycaemia can be treated easily, the current targets for treatment are not supported by robust evidence. A new study has demonstrated that maintaining blood levels of glucose of at least $2.6 \mathrm{mmol} / \mathrm{l}$ is safe and effectively prevents neurological impairment in neonates with hypoglycaemia.

"It has been known for decades that hypoglycaemia can cause brain injury in the newborn, but the question of how low is too low in which babies remains unresolved," explains corresponding author Jane Harding (University of Auckland, New Zealand). To answer this question, Harding and her colleagues recruited a cohort of 404 newborn babies who were at risk of hypoglycaemia (risk factors included maternal diabetes mellitus, birth at $<37$ gestational weeks, low birth weight and high birth weight). All the participants had a gestational age of at least 35 weeks. The neonates were all managed in a consistent way, and were treated according to current standard guidelines. The neonates underwent regular glucose monitoring for $24 \mathrm{~h}$ or $48 \mathrm{~h}$ after birth, or until there were no ongoing clinical concerns, and some received continuous glucose monitoring (these results were blinded to the treating clinicians). The cohort contained neonates who experienced hypoglycaemia and were treated, neonates who did not develop hypoglycaemia and neonates with low interstitial glucose concentrations who were not treated as they were only detected during continuous glucose monitoring. "This cohort provided an ideal group to determine the relationship between neonatal glucose concentrations and developmental outcomes," says Harding.

Regular glucose monitoring identified 216 neonates with hypoglycaemia (defined as blood levels of glucose $<2.6 \mathrm{mmol} / \mathrm{l}$ ). These babies were treated with any combination of additional feeding, buccal dextrose gel or intravenous dextrose to maintain their blood glucose levels above $2.6 \mathrm{mmol} / \mathrm{l}$. At 2 years of age, all the participating children underwent tests to assess their neurological development. Executive function was tested with four tasks to assess inhibitory control, reverse categorization and attentional flexibility. The children were also assessed using the Bayley Scales of Infant Development III and underwent vision screening and global motion perception testing. Caregivers also filled out questionnaires about the home environment, the child's health and their executive function.

The researchers did not find any notable differences in neurological outcomes between the neonates who underwent treatment to maintain blood levels of glucose of at least $2.6 \mathrm{mmol} / \mathrm{l}$ and the neonates who did not develop hypoglycaemia. "This is the first robust evidence that a blood glucose level of at least $2.6 \mathrm{mmol} / \mathrm{l}$ is a safe treatment threshold for neonatal hypoglycaemia," states Harding. Of note, neonates with low
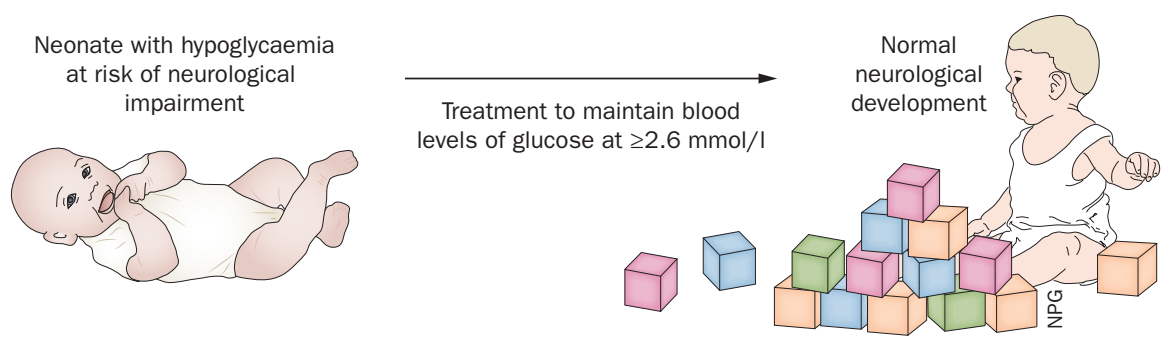

blood glucose levels that were only detected on continuous blood glucose monitoring did not experience impaired neurological development. This finding indicates that intermittent blood glucose monitoring is sufficient for managing neonates at risk of hypoglycaemia.

Interestingly, children who had impaired neurodevelopment tended to have higher blood levels of glucose than the children who did not have neurological impairment. These children also spent more time with blood levels of glucose outside the central range $(3.0-4.0 \mathrm{mmol} / \mathrm{l})$ in the first $48 \mathrm{~h}$ after birth than children with normal neurological development. "This finding needs confirmation, but does raise the question of whether high or fluctuating glucose concentrations in the neonatal period might contribute to adverse outcomes," explains Harding.

Neurological impairment might become evident after 2 years of age, as hypoglycaemia could affect skills that emerge at later stages of development. Follow-up of the cohort is in progress, and neurological development has already been assessed at the age of 4.5 years. "Analysis of these results might help to confirm or refute the findings of this study," says Harding. "In addition, these findings could form the basis for the design of new randomized trials to determine whether treating babies at different thresholds might alter neurodevelopmental outcomes."

The investigators conclude that treating neonates who have hypoglycaemia to maintain their blood levels of glucose $\geq 2.6 \mathrm{mmol} / \mathrm{l}$ effectively prevents neurological impairment. However, they note that further research is needed to confirm these findings.

Claire Greenhill

Original article McKinlay, C. J. D. et al. Neonatal glycemia and neurodevelopmental outcomes at 2 years. N. Engl. J. Med. 373, 1507-1518 (2015) 